\title{
Novos aditivos de secagem para concretos refratários: fibras poliméricas de alto desempenho
}

\author{
(New drying additives for refractory castables: \\ high performance polymeric fibers)
}

\author{
R. Salomão ${ }^{1}$ L. R. M. Bittencourt ${ }^{2}$, V. C. Pandolfelli ${ }^{1}$ \\ ${ }^{I}$ Departamento de Engenharia de Materiais - DEMa \\ Universidade Federal de S. Carlos - UFSCar \\ Rod. Washington Luiz, km 235, C.P. 676, S. Carlos, SP 13565-905 \\ ${ }^{2}$ Centro de Pesquisas - Magnesita S.A. \\ Praça Louis Ensch, 240, Contagem, MG 32210-050 \\ pers@iris.ufscar.br,vicpando@power.ufscar.br
}

\begin{abstract}
Resumo
Fibras poliméricas têm sido sistematicamente incorporadas às formulações de concretos refratários com o objetivo de diminuir os riscos de explosão durante o processo de secagem. Após a fusão das fibras, canais permeáveis são formados, reduzindo a pressão de vapor e aumentando a velocidade de secagem. As fibras comumente utilizadas para esse fim são de polipropileno e podem gerar aumentos de permeabilidade em temperaturas por volta de $165^{\circ} \mathrm{C}$. Para otimizar o desempenho desses materiais é necessário que essa temperatura seja reduzida, preferencialmente para abaixo de $100^{\circ} \mathrm{C}$. Como não há grande disponibilidade de produtores de fibras com esse requisito, um sistema de fiação e uma nova geração de fibras poliméricas foram desenvolvidos baseados em uma criteriosa seleção de polímeros e copolímeros, bem como em suas composições químicas e propriedades físicas. Fibras com geometria similar às comerciais foram produzidas e sua atuação como aditivo de secagem foi avaliada, apresentando desempenho consideravelmente superior.
\end{abstract}

Palavras-chave: concretos refratários, secagem, fibras poliméricas.

\begin{abstract}
Polymeric fibers have been systematically added to the refractory castables formulations in order to reduce the risks of explosive spalling during the drying and first heat-up process. After fibers melt, permeable paths are generated, reducing the vapor pressure and increasing the drying rate. The polypropylene fibers are traditionally employed in this technique and modify permeability at $165{ }^{\circ} \mathrm{C}$. To optimize the performance of these fibers, their melting point must be reduced bellow to $100{ }^{\circ} \mathrm{C}$. As there is not great availability of fibers with this requirement, a melt-spinning equipment and a new generation of fibers were developed, based on a careful choice of polymers and copolymers, as well as their composition and physical and chemical properties. Fibers with geometry similar to the conventional ones were produced and their performance as drying additives were evaluated, presenting better results than the conventional polypropylene ones.

Keywords: refractory castables, drying polymeric fibers.
\end{abstract}

\section{INTRODUÇÃO}

Fibras poliméricas como aditivos de secagem

Concretos refratários são materiais densos e de baixa permeabilidade. Devido a isso, seu processo de secagem e aquecimento inicial enfrenta sérios obstáculos. A dificuldade que o vapor de água encontra para deixar sua estrutura pode levar a uma explosão se a pressão desenvolvida atingir o limite de resistência mecânica do material. Para reduzir os riscos de explosão e diminuir o tempo de secagem, fibras poliméricas têm sido incorporadas às formulações de concretos refratários como aditivos de secagem [1-3].

Uma vez misturadas aos componentes do concreto, o espaço ocupado por elas é preservado durante o processo de cura. No primeiro aquecimento, quando a temperatura atinge o ponto de fusão do polímero, esses espaços se tornam regiões de elevada permeabilidade, permitindo a liberação do vapor pressurizado. Para que esse mecanismo atue de forma eficaz, dois requerimentos principais devem ser satisfeitos: 1) a geometria das fibras e o teor adicionado às formulações devem ser otimizados para promover um aumento de permeabilidade elevado 
$[4,5]$ e 2) a faixa de temperatura em que ele ocorre deve ser tão baixa quanto possível para evitar que o vapor de água atinja temperaturas superiores a $100^{\circ} \mathrm{C}[6]$.

$\mathrm{O}$ primeiro requerimento pode ser satisfeito pela otimização da geometria inicial das fibras, das condições de mistura e da quantidade adicionada. As relações desses parâmetros com o processo de secagem e as condições otimizadas foram recentemente descritas [3-5]. Já o segundo, foi alvo de algumas publicações focadas em diferentes tipos de fibras comerciais [6]. Vários tipos, como fibras de polipropileno, de poliéster e aramídicas, adaptadas de outras aplicações (têxteis) tiveram seu desempenho como aditivo de secagem avaliado. Entre elas, as de polipropileno apresentaram os melhores resultados devido ao menor ponto de fusão $\left(165^{\circ} \mathrm{C}\right.$, em comparação com as de poliéster, $235^{\circ} \mathrm{C}$, e aramídicas, que somente se decompõe acima de $350^{\circ} \mathrm{C}$ ).

Mudar a geometria das fibras empregadas em uma certa formulação é algo relativamente fácil devido ao grande número de produtos e produtores existente. Por outro lado, mudar a composição das fibras é um processo mais complexo, pois envolve alterações na química dos polímeros utilizados e nas condições de processamento. Essas dificuldades operacionais e o fato do consumo de fibras para aditivação de secagem ser muito menor que seu uso têxtil podem ser apontados como as principais razões para que apenas fibras comerciais sejam empregadas na indústria de refratários. Como resultado, a otimização das propriedades físico-químicas das fibras para atuar na secagem ainda está longe de ser atingida.

O processo de fiação de polímeros fundidos e suas peculiaridades

O processo mais comum de manufatura de fibras sintéticas é conhecido como fiação do fundido. Ele consiste em fundir um determinado polímero em um extrusora e forçá-lo a fluir através de uma matriz contendo inúmeros pequenos orifícios (geralmente, mais de 200 e com diâmetros abaixo de 0,1 $\mathrm{mm}$ ). Logo após a extrusão, os filamentos se solidificam com o resfriamento e são estirados para atingir elevada orientação molecular e, devido a isso, elevada resistência mecânica e estabilidade térmica. Dispositivos de mecânica de precisão permitem atingir um delicado equilíbrio entre as quantidades de polímero extrudado e estirado. Filamentos com diâmetros de até $2 \mu \mathrm{m}$ (micro-fibras) e com seção transversal de diversas formas (circular, quadrada, cruz-demalta, circular oca) podem ser produzidos [7].

Comparada com outros processos, a fiação do fundido apresentou um significativo crescimento nas últimas décadas devido à sua praticidade, ausência de voláteis e elevada produtividade (algumas linhas podem atingir velocidades de até $400 \mathrm{~km} / \mathrm{h}$ ). Para ser fiado por esse método, tipos especiais de polímeros devem ser empregados. Esses materiais são sintetizados com cuidadosa composição e distribuição de peso molecular para assegurar ao fundido baixa viscosidade e, ao mesmo tempo, elevada elasticidade. Uma vez que o polímero apresente essas características, praticamente qualquer tipo de material pode ser convertido na forma de fibra. No entanto, devido à grande variedade de materiais poliméricos, as propriedades das fibras obtidas podem variar drasticamente e alguns tipos podem não ser necessariamente úteis [7].

\section{Produção dos novos tipos de fibras}

Os principais tipos de fibras têxteis, poliéster (PET), poliamidas (Nylons) e polipropileno (PP), foram desenvolvidos para apresentar elevada resistência mecânica e estabilidade térmica e química. As bases do processo de estabilização são a introdução de agentes anti-oxidantes nas formulações das fibras e o aumento do grau de cristalinidade dos polímeros (maior porcentagem de fases cristalinas e cristais mais perfeitos) por meio da orientação molecular durante o estiramento das fibras. Como conseqüência desses tratamentos, o ponto de fusão das fibras e sua resistência termo-oxidativa são aumentados (em alguns casos, em mais de $50{ }^{\circ} \mathrm{C}$ ) [7].

Esses tratamentos são essenciais às aplicações têxteis (como roupas, carpetes e não-tecidos), mas atuam desfavoravelmente quando a aplicação das fibras é como aditivo de secagem. Devido a isso, para melhorar seu desempenho durante secagem, as fibras devem ser altamente susceptíveis aos efeitos da temperatura. Essa consideração leva a fibras com baixo ponto de fusão (próximo a $100{ }^{\circ} \mathrm{C}$ ) e facilmente degradáveis pela temperatura, apresentando simultaneamente, geometria adequada e resistência mecânica mínima para resistir às condições de mistura.

Este trabalho demonstrou que o desenvolvimento de fibras especificamente projetadas para atuar na secagem de concretos é possível e pode trazer importantes vantagens competitivas aos produtores de refratários. Uma cuidadosa escolha de polímeros e copolímeros foi feita com base em seus comportamentos térmicos (baixo ponto de fusão), propriedades reológicas (capacidade de formar fibras) e custos envolvidos. Como os tipos comerciais de polímeros convencionalmente empregados em fiação do fundido não apresentam características adequadas, três diferentes materiais, oriundos de outras aplicações, foram selecionados para a produção de fibras: 1) polipropileno reciclado a partir de filmes de embalagem (PPr), 2) polietileno de baixa densidade linear (PEBDL) e 3 ) copolímero polietileno de baixa densidade e acetato de vinila. Um equipamento de fiação foi desenvolvido e fibras foram produzidas em geometrias similares às comerciais. Finalmente, o desempenho dessa nova geração de aditivos de secagem foi comparado com o das fibras de polipropileno comumente empregadas, apresentando resultados significativamente superiores. 


\section{MATERIAIS E METODOS}

\author{
Equipamento defiação, fiação dofundidoe caracterização \\ das fibras
}

A Fig. 1 mostra detalhes do equipamento de fiação desenvolvido no grupo de pesquisa onde este trabalho foi desenvolvido [7]. Ele consiste em uma extrusora com elementos de aquecimento (até $300{ }^{\circ} \mathrm{C}$ ) para manter a temperatura constante durante o processo. $\mathrm{O}$ polímero fundido é forçado a fluir através de uma matriz com orifícios de diâmetro da ordem de $0,1 \mathrm{~mm}$. Acoplado à extrusora, há um coletor que estira os filamentos. As velocidades de extrusão e estiramento foram balanceadas para produzir fibras com diâmetros similares àquelas comerciais. Após a fiação e estiramento, as fibras foram cortadas em segmentos de $3 \mathrm{~mm}$ de comprimento.

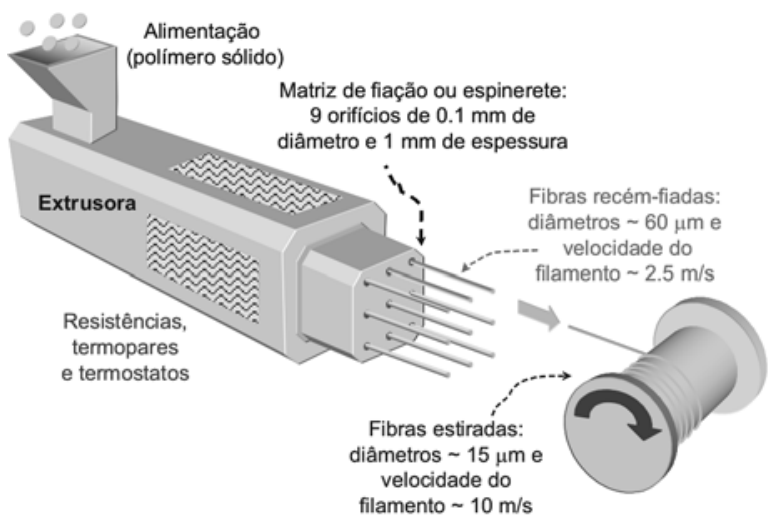

Figura 1: Equipamento de fiação desenvolvido [7]. [Figure 1: Melt-spinning equipment developed [7].]

Os novos tipos de fibras e aquelas convencionais de polipropileno foram caracterizadas por calorimetria exploratória diferencial (CED ou differential scanning calorimetry, DSC) para determinação de seus pontos de fusão. Os testes foram conduzidos entre temperatura ambiente $\left(25^{\circ} \mathrm{C}\right)$ e a de fusão dos polímeros, sob uma taxa de aquecimento de $10{ }^{\circ} \mathrm{C} / \mathrm{min}$ e atmosfera de ar sintético. Os diâmetros e comprimentos das fibras, antes e após a mistura no concreto, foram medidos utilizandose um microscópio ótico Leica DMR (aumento de 100 vezes) e uma câmera digital MavicaFD, respectivamente. Em ambos os casos foi utilizado o analisador de imagens Image-Pró 4.1.1.1 e para cada tipo de fibra, pelo menos 800 medidas foram realizadas.

\section{Preparação das amostras}

Foi utilizada uma formulação de concreto refratário autoescoante, elaborada segundo o modelo de empacotamento de partículas de Andreasen com coeficiente q igual a 0,21 , composta de uma matriz fina (24\%peso, Dp $<100 \mu \mathrm{m}$, Almatis, EUA) e agregados (76\%peso, Dp $\geq 100 \mu \mathrm{m}$, Elfusa, Brasil) e contendo $98 \%$ peso de alumina e $2 \%$ peso de cimento de aluminato de cálcio (CA14M, Almatis, EUA). Um teor de 4,5\%peso de água foi adicionado à composição para mistura e hidratação do cimento. Os diferentes tipos de fibras foram previamente misturados ao pó seco em um teor volumétrico de $0,36 \%$.

Após a mistura, as composições foram moldadas na forma de cilindros de $70 \mathrm{~mm}$ de diâmetro por $25 \mathrm{~mm}$ de altura, para a avaliação da permeabilidade, e de $40 \mathrm{~mm}$ de diâmetro por $40 \mathrm{~mm}$ de altura, para os testes de secagem e explosão. Durante a moldagem, termopares tipo K foram inseridos nas amostras logo abaixo da superfície e à meia altura do cilindro. Essas amostras foram curadas a $8{ }^{\circ} \mathrm{C}$, por 15 dias, em uma câmara climatizada (Vötsch 2020). As amostras para testes de secagem e explosão foram ensaiadas logo após esse período; aquelas destinadas à avaliação da permeabilidade foram secas em sílica-gel por $96 \mathrm{~h}$, a $8^{\circ} \mathrm{C}$.

Medidas de permeabilidade e comportamento fluidodinâmico e de secagem

A magnitude do aumento de permeabilidade gerado pelos diferentes tipos de fibras foi obtida por medidas realizadas em amostras verdes (secas a $8^{\circ} \mathrm{C}$ ) e queimadas (a $900{ }^{\circ} \mathrm{C}$, por $6 \mathrm{~h}$ ). As constantes de permeabilidade Darcyana $\left(k_{1}\right)$ e não-Darcyana $\left(k_{2}\right)$ foram calculadas por meio do ajuste polinomial de dados de pressão e velocidade de escoamento do ar à Equação de Forchheimer (A), expressa para fluidos compressíveis como

$$
\frac{P_{i}^{2}-P_{0}^{2}}{2 P_{0} L}=\frac{\mu}{k_{1}} v_{s}+\frac{\rho}{k_{2}} v_{s}^{2}
$$

onde Pi e Po são as pressões absolutas do ar na entrada e na saída da amostra, Vs e a velocidade do fluido, L é a espessura da amostra e $\mu$ e $\rho$ são a viscosidade e a densidade do fluido (ar, a $25^{\circ} \mathrm{C}$ ). O termo linear da Equação de Forchheimer $\left(\mu \mathrm{v}_{\mathrm{s}} / \mathrm{k}_{1}\right)$, que representa as perdas de energia relacionadas ao atrito, prevalece em baixas velocidades. Já o termo quadrático $\left(\rho v_{\mathrm{s}}^{2} / \mathrm{k}_{2}\right)$ define a contribuição da inércia e turbulência devido à tortuosidade do meio na queda de pressão e é mais relevante em altas velocidades, como no caso da secagem. Os valores das constantes de permeabilidade foram obtidos pelo ajuste dos pontos experimentais de $\left(\mathrm{P}_{\mathrm{i}}^{2}-\right.$ $\left.\mathrm{P}_{0}{ }^{2}\right) / 2 \mathrm{P}_{0} \mathrm{~L}$ em função de $\mathrm{v}_{\mathrm{s}}$ à Equação $\mathrm{A}$, utilizando-se o métodos dos mínimos quadráticos. Esses valores podem variar significativamente durante as etapas da secagem e assim indicar a ativação de diferentes fenômenos microestruturais [6]. 
O comportamento fluidodinâmico das formulações foi caracterizado por meio da técnica de permeametria de ar quente, de $25^{\circ} \mathrm{C}$ a $400{ }^{\circ} \mathrm{C}$, sob uma taxa de aquecimento de $5{ }^{\circ} \mathrm{C} / \mathrm{min}$. Nessa técnica, um fluxo de ar, gerado por um gradiente de pressão $(0,3 \mathrm{MPa})$ é forçado a percolar a amostra de concreto. Sob esse gradiente de pressão elevado, a velocidade do fluxo de ar se torna altamente sensível a rearranjos do meio poroso, evidenciando variações de permeabilidade provocadas pela temperatura, sem necessidade de tratamentos térmicos prévios [6].

Os ensaios de secagem foram feitos em um aparato termogravimétrico desenvolvido pelo Grupo de Pesquisa dos autores, que permite registrar simultaneamente as variações de massa e o perfil de temperatura dentro do forno e na superfície da amostra. Uma taxa de aquecimento de $10{ }^{\circ} \mathrm{C} / \mathrm{min}$ foi aplicada entre $25{ }^{\circ} \mathrm{C}$ e $600{ }^{\circ} \mathrm{C}$. As variações de massa foram acompanhadas por meio da derivada do parâmetro $\mathrm{W}$ em relação ao tempo, que avalia a fração cumulativa de massa perdida durante o aquecimento, em relação ao teor inicial de água na amostra (Equações B e C),

$$
\begin{gathered}
W(\%)=100 \times \frac{M_{0}-M}{M_{0}-M_{f}} \\
\left(\frac{d W}{d t}\right)_{i}=\frac{W_{i+1}-W_{i-1}}{t_{i+1}-t_{i-1}}
\end{gathered}
$$

onde: M é a massa instantânea registrada no tempo $t_{i}$, durante $\mathrm{o}$ aquecimento, $\mathrm{M}_{0}$ é a massa inicial e $\mathrm{M}_{\mathrm{f}}$ a massa final da amostra [8].

\section{RESULTADOS E DISCUSSÃO}

Medidas de permeabilidade e geometria das fibras após a mistura com o concreto

As medidas de comprimentos e diâmetros das fibras realizadas após a mistura com o concreto indicaram que tanto os novos tipos de fibras como as convencionais de polipropileno sofreram um mesmo nível de dano (principalmente redução de comprimento) devido ao cisalhamento e abrasão, resultando em fibras com geometrias

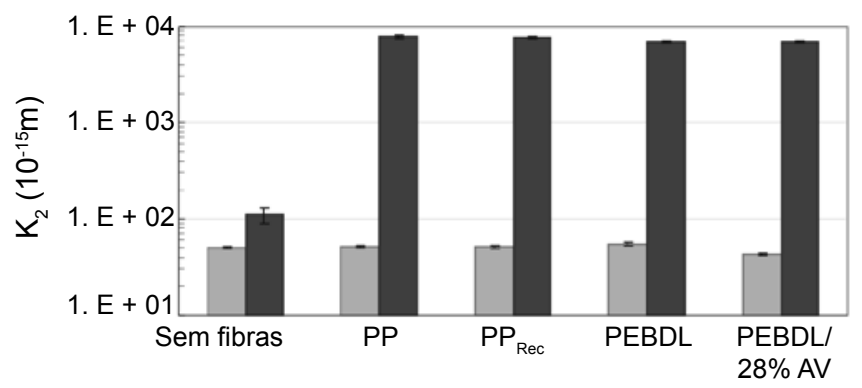

Figura 2: Medidas de permeabilidade para amostras contendo fibras poliméricas.

[Figure 2: Permeability measurements for polymeric fiber containing castables].

Tabela I - Características das fibras desenvolvidas.

[Table I - Characteristics of the developed fibers].

\begin{tabular}{ccccc}
\hline $\begin{array}{c}\text { Fibras } \\
\text { Composição }\end{array}$ & $\begin{array}{c}\text { PP } \\
\text { Polipropileno / } \\
\text { Polypropylene }\end{array}$ & $\begin{array}{c}\text { Polipropileno reciclado / } \\
\text { Recycled polypropylene }\end{array}$ & $\begin{array}{c}\text { PEBDL } \\
\text { Polietileno de baixa } \\
\text { densidade linear / } \\
\text { Linear low density } \\
\text { polyethylene } \\
\text { (LLDPE) }\end{array}$ & $\begin{array}{c}\text { PEBD/AV } \\
\text { Copolímero 28\% } \\
\text { peso acetato de vinila / } \\
\text { Copolymer LDPE / } \\
\text { Vinyl acetate } \\
\text { (VA/LDPE) }\end{array}$ \\
\hline Aplicação de origem & $\begin{array}{c}\text { Carpetes e } \\
\text { não-tecidos }\end{array}$ & & Filmes para embalagens & \\
\hline Fusão $\left({ }^{\circ} \mathrm{C}\right)$ & 165 & 163 & 107 & 75 \\
\hline Densidade $\left(\mathrm{g} / \mathrm{cm}^{3}\right)$ & 0,91 & 0,91 & 0,88 & 0,89 \\
\hline $\begin{array}{c}\text { Diâmetro }(\mu \mathrm{m}) \\
\text { Comprimento } \\
\text { inicial }(\mathrm{mm})\end{array}$ & $15 \pm 0,89$ & $20 \pm 1,56$ & $18 \pm 1,23$ & $24 \pm 1,33$ \\
\hline $\begin{array}{c}\text { Comprimento } \\
\text { após mistura }(\mathrm{mm})\end{array}$ & $3,33 \pm 0,99$ & $4,58 \pm 0,87$ & $3,89 \pm 0,98$ & $3,98 \pm 0,98$ \\
\hline
\end{tabular}


similares (Tabela I). A permeabilidade das amostras verdes não foi afetada pela adição de fibras. Após o tratamento térmico, no entanto, aumentos de permeabilidade similares e de até duas ordens de magnitude nos valores de $\mathrm{k}_{2}$ foram observados (Fig. 2) para as amostras contendo fibras.

Esses resultados são coerentes com sistemas similares descritos na literatura $[4,6]$. Uma vez que diferentes tipos de fibras sejam adicionados à uma mesma formulação de concreto, em um mesmo teor volumétrico, com geometrias similares e processadas da mesma forma, níveis de permeabilidade similares podem ser obtidos após tratamento térmico. Desse modo, pode-se afirmar que os novos tipos de fibras possuem propriedades mecânicas adequadas para resistir ao cisalhamento e abrasão durante a mistura e também que, após esse processo, sua geometria permite gerar aumentos de permeabilidade tão significativos quanto aqueles proporcionados pelas fibras convencionais.

\section{Comportamento fluidodinâmico e de secagem}

A secagem de concretos refratários geralmente apresenta três estágios principais: evaporação (até $100^{\circ} \mathrm{C}$ ), ebulição $\left(100-500^{\circ} \mathrm{C}\right)$ e decomposição de fases hidratadas dos ligantes
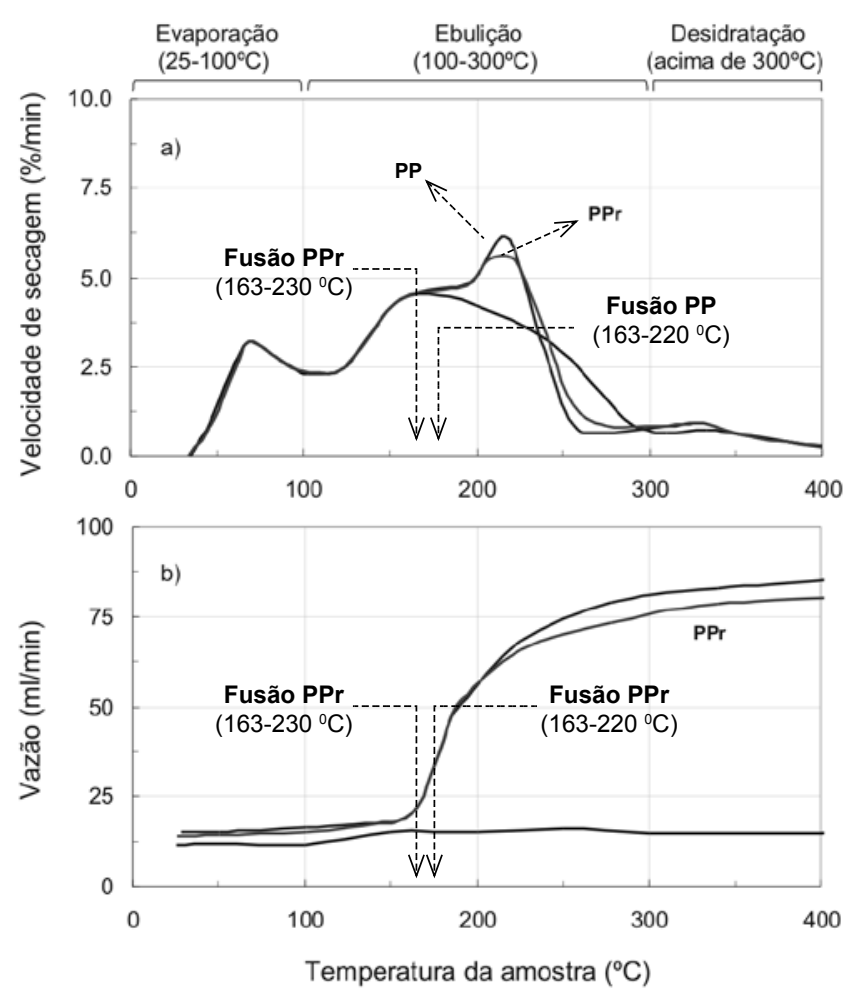

Figura 3: Comportamento de a) secagem e explosão e b) fluidodinâmico para amostras de concretos com fibras de polipropileno convencionais e produzidas a partir de polímero reciclado.

[Figure 3: [a] Drying and explosive spalling and b) fluidynamic behavior for castables containing polypropylene and recycled polypropylene fibers]. empregados (acima de $250{ }^{\circ} \mathrm{C}$, para o cimento de aluminato de cálcio). No entanto, durante a ebulição, a pressão de vapor da água pode superar a resistência mecânica do material causando explosões. Recentemente foi demonstrado que com a adição de fibras poliméricas adequadas à formulação de concreto, a permeabilidade do material pode ser aumentada controladamente evitando a explosão e aumentando a taxa de secagem [1]. Esse aumento de permeabilidade ocorre quando as fibras se fundem e se tornam fluidas o suficiente para serem deformadas pelo vapor pressurizado.

Para as amostras contendo fibras convencionais de polipropileno, esse comportamento pode ser identificado como um aumento localizado da taxa de secagem (Fig. 3a) e na vazão da permeametria de ar quente (Fig. 3b), por volta de $165-170{ }^{\circ} \mathrm{C}$. A amostra com fibras de polipropileno reciclado apresentou comportamentos de secagem e fluido dinâmico equivalentes. Essa similaridade pode ser atribuída a dois fatores: 1) apesar do processo de reciclagem que o polímero foi submetido, sua temperatura de fusão não foi afetada significativamente, permanecendo muito próxima à do material convencional e 2) como mostrado na Fig. 2, as fibras de material reciclado proporcionaram aumentos de permeabilidade: geometria e resistência à mistura adequadas.

$\mathrm{O}$ fato de fibras produzidas a partir de matérias primas menos nobres poderem apresentar desempenho muito similar àquelas convencionais, pode ser visto como uma oportunidade de reduzir
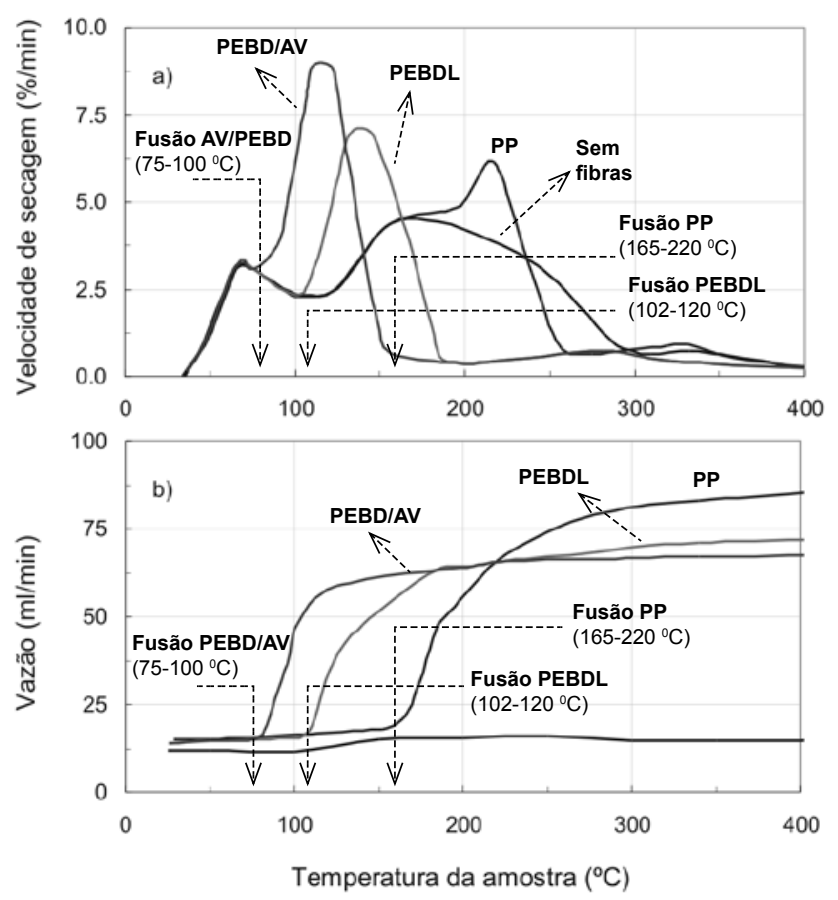

Figura 4: Comportamento de a) secagem e explosão e b) fluidodinâmico para amostras de concretos com fibras de polipropileno, PEBDL e $\mathrm{PEBD} / \mathrm{AV}$.

[Figure 4: a) Drying and explosive spalling and b) fluidynamic behavior for castables containing polypropylene, LLDPE and LDPE/VA fibers.] 
custos envolvidos tanto na formulação dos concretos quanto na produção das fibras. Polipropileno reciclado usualmente é mais barato que o polímero virgem, pode ser processado utilizandose os mesmos equipamentos ou com pequenas alterações e representa uma alternativa ecologicamente aceitável à disposição desses materiais no meio ambiente.

Para as fibras de PEBDL e PEBDL/AV, os aumentos de taxa de secagem (Fig. 4a) e permeabilidade (Fig. 4b) ocorreram em menores temperaturas $\left(110{ }^{\circ} \mathrm{C}\right.$ e $75-80{ }^{\circ} \mathrm{C}$, respectivamente). Esse fato está associado às menores temperaturas de fusão desses materiais permitindo que estruturas altamente permeáveis fossem formadas nos concretos antes do início da pressurização do vapor de água. Como resultado, após o início da ebulição $\left(110^{\circ} \mathrm{C}\right)$, foram geradas taxas de secagem muito maiores que as observadas nos concretos sem fibras e com fibras de polipropileno. A redução da temperatura em que o valor máximo da taxa de secagem ocorre pode ser vista como uma outra importante conseqüência: com a diminuição da faixa de temperatura em que a secagem ocorre, reduz-se também os riscos de pressurização da estrutura e explosões [8].

\section{CONCLUSÕES}

Comparadas às fibras de polipropileno tradicionalmente empregadas como aditivos de secagem em concretos refratários, os novos tipos de fibras desenvolvidos apresentaram desempenhos bastante adequados. As fibras produzidas a partir de polipropileno reciclado proporcionaram um comportamento similar àquele gerado pelas fibras convencionais. Esse fato pode ser visto como uma boa oportunidade de se reduzir os custos de produção das formulações de concreto e de produção das fibras. Já as de PEBDL e PEBD/AV apresentaram um desempenho bastante superior como aditivo de secagem. Devido a seus menores pontos de fusão, essas fibras geraram aumentos de permeabilidade em temperatura da ordem de $80-110{ }^{\circ} \mathrm{C}$, aumentando significativamente a taxa de secagem e reduzindo os riscos de explosão. Esses resultados evidenciam a necessidade de otimização das fibras poliméricas convencionais empregadas como aditivo de secagem em concretos refratários e ressaltam as grandes possibilidades de pesquisa e desenvolvimento tecnológico nessa área. Pela originalidade e impacto desta pesquisa, as fibras desenvolvidas e seu emprego estão em processo de patenteamento.

\section{AGRADECIMENTOS}

Os autores agradecem à FAPESP, à Alcoa Alumínio e à Magnesita S.A. pelo suporte fornecido a este trabalho.

\section{REFERÊNCIAS}

[1] R. Salomão, V. C. Pandolfelli, J. Tech. Assoc. Refr. Jpn. 24, 2 (2004) 83-87.

[2] C. M. Peret, R. Salomão, V. C. Pandolfelli, J. Tech. Assoc. Refr. Jpn. 24, 2 (2004) 88-92.

[3] R. Salomão, V. C. Pandolfelli, "Modifying the drying behavior of refractory castables by polymeric fiber addition", Am. Cer. Soc. Bull. (2005) submetido.

[4] R. Salomão, V. G. Domiciano, C. S. Isaac, R. G. Pileggi, V. C. Pandolfelli, Am. Cer. Soc. Bull. 85, 1 (2004) 93019308.

[5] R. Salomão, M. A. Zambon, V. C. Pandolfelli, "Polymeric Fiber-Containing Refractory Castables: Fiber Geometry and Permeability", Am. Ceram. Soc. Bull. (2005) submetido.

[6] M .D. M. Innocentini, C. Ribeiro, R. Salomão, F. A. Cardoso, R. Rettore, L. R. M. Bittencourt, V. C. Pandolfelli, Am. Ceram. Soc. Bull. 81, 8 (2002) 65-68.

[7] Y. Murase, A. Nagai, Melt Spinning, in Advanced Fiber Spinning Technology, Ed. Nakajima, Cambridge, Inglaterra (1994) 25-54.

[8] M. D. M. Innocentini, F. A. Cardoso, M. M. Akyioshi, V. C. Pandolfelli, J. Am. Ceram. Soc. 86, 7 (2003) 1146-48. (Rec. 25/05/2005, Ac.21/10/2005) 